\title{
Surfaces
}

\section{Antoine Berman, Pour une critique des traductions : John Donne, Paris, Gallimard, 1995, 276 p.}

\section{Rachel Bouvet}

Volume 5, 1995

URI : https://id.erudit.org/iderudit/1065008ar

DOI : https://doi.org/10.7202/1065008ar

Aller au sommaire du numéro

Éditeur(s)

Les Presses de l’Université de Montréal

ISSN

1188-2492 (imprimé)

1200-5320 (numérique)

Découvrir la revue

Citer ce compte rendu

Bouvet, R. (1995). Compte rendu de [Antoine Berman, Pour une critique des traductions : John Donne, Paris, Gallimard, 1995, 276 p.] Surfaces, 5.

https://doi.org/10.7202/1065008ar

Ce document est protégé par la loi sur le droit d'auteur. L'utilisation des services d'Érudit (y compris la reproduction) est assujettie à sa politique d'utilisation que vous pouvez consulter en ligne.

https://apropos.erudit.org/fr/usagers/politique-dutilisation/ 


\section{Compte rendu}

\section{Antoine Berman : Pour une critique des traductions : John Donne}

Rachel Bouvet

Université de Montréal

Surfaces Vol.V.04 (v.1.0F - 19/12/1995)

Tout texte reste la propriété de son auteur. Néanmoins, SURFACES demande d'être citée à l'occasion de toute autre publication du texte en question.

ISSN: $1188-2492$

\section{Antoine Berman, Pour une critique des traductions: John Donne, Paris, Gallimard, 1995, 276 p.}

Dans cet ouvrage posthume, écrit pendant les trois derniers mois précédant sa mort, Antoine Berman propose un nouveau genre de critique littéraire: la critique des traductions. S'appuyant sur le concept de critique de Benjamin, l'auteur entend combler une lacune de la critique actuelle, qui étudie les textes étrangers "soit dans leur langue, soit dans une version française en 'oubliant' qu'il s'agit d'une version" (p. 41). Sa démarche consiste à replacer les réflexions théoriques sur la traduction littéraire au sein de la réflexion sur la littérature et sur la façon dont elle se donne à lire. Il insiste d'ailleurs sur la parenté entre l'acte critique et l'acte de traduction en affirmant que la lecture du texte et son étude critique sont des étapes indispensables du processus de traduction.

Inspiré par l'herméneutique philosophique de Ricœur et l'herméneutique littéraire de Jauss, le modèle établi se démarque considérablement des travaux effectués jusque là sur la traduction. L'auteur distingue les analyses faites en l'absence de méthodologie précise, qui s'emploient à relever les écarts entre l'original et la traduction ou à examiner de manière érudite l'œuvre d'un traducteur, des analyses possédant une forme forte, "qui se réfléchit elle-même, thématise sa spécificité et, ainsi, produit sa méthodologie" (p. 45). Ces dernières sont soit de type "source-oriented", 
comme celles de Meschonnic, qui mettent en évidence les partis pris idéologiques des traducteurs, les modes esthétiques, etc., soit de type "target-oriented", comme les analyses descriptives à orientation sociocritique de l'école de Tel-Aviv (Toury en particulier), qui s'intéressent aux normes, au polysystème culturel dans lequel l'œuvre traduite s'intègre, ayant tendance à privilégier un objet d'étude en particulier: la traductionadaptation. Le projet de Berman s'inscrit quant à lui dans une toute autre perspective, qui pourrait être qualifiée de "reader-oriented". La méthode d'analyse préconise en effet une succession d'actes de lecture différents, allant de la lecture du texte traduit à la lecture comparée avec le texte original, en passant par les lectures collatérales, la mise à jour des principes interprétatifs du traducteur et des premiers critiques de la traduction, etc.

La première étape de la critique des traductions consiste à effectuer une lecture de la traduction. Celle-ci n'est pas considérée à priori comme un texte second, possédant moins de valeur que l'original. Comme le souligne Berman,

La première lecture reste encore, inévitablement, celle d'une "œuvre étrangère" en français. La seconde la lit comme une traduction, ce qui implique une conversion du regard. Car, comme il a été dit, on n'est pas naturellement lecteur de traductions, on le devient. (p. 65)

Le fait de résister à la compulsion de comparaison, d'avoir avec le texte traduit une certaine intimité, non troublée par des éléments provenant du texte original, permet au critique d'avoir des impressions de lecture, de repérer par exemple certaines zones textuelles problématiques, ou encore d'évaluer la cohérence de l'ensemble. Cette première lecture, indispensable pour pouvoir mener à bien le travail d'analyse de la traduction, est suivie de la lecture de l'original, lieu d'une pré-analyse textuelle axée sur le repérage de certaines zones problématiques, des traits stylistiques, des rythmes, des mots-clefs, travail que le traducteur est censé avoir fait lui aussi. Puis, le critique effectue des lectures collatérales (autres œuvres de l'auteur, du traducteur, essais critiques, etc.) et prend connaissance de l'étayage de la traduction (préface, notes, glossaires, etc.), toujours dans le but de préparer la confrontation entre la traduction et l'original. Enfin, il sélectionne des exemples stylistiques significatifs et, à partir de sa propre interprétation de l'œuvre, identifie certains "passages de l'original qui, pour ainsi dire, sont les lieux où [l'œuvre] se condense, se représente, se signifie ou se symbolise" (p. 70).

Dans un troisième temps, c'est le traducteur lui-même qui fait l'objet de recherches. L'interrogation porte sur son identité, sur sa position traductive, pouvant être reconstituée à partir de ses écrits sur la traduction, sur son projet de traduction, étant donné qu'un choix a été fait parmi les différents textes d'un auteur, parmi les différentes manières de traduire, sur l'horizon du traducteur, autrement dit l'ensemble des discours présents à l'époque de la traduction ayant porté de près ou de loin sur cette activité. Le rôle du critique n'est pas de remettre en cause le projet de traduction mais d'examiner de quelle façon il a été réalisé. En ce qui concerne l'horizon du traducteur, il faut rappeler que Berman s'est intéressé à plusieurs reprises 
aux discours sur la traduction. Son livre L'épreuve de l'étranger 1 examine en détail les écrits de l'Allemagne romantique, qui font d'ailleurs partie de ce qu'il nomme ailleurs "les discours traditionnels" $\underline{2}$. Étudiés dans une perspective plus large, embrassant l'ensemble des discours tenus sur la traduction dans les différentes disciplines, les discours traditionnels sont comparés à d'autres types de discours: les discours objectifs sectoriels, formulés par des linguistes, des poéticiens, des comparatistes; les discours généraux, où de véritables théories de la traduction sont élaborées; les discours d'expérience, abordant la traduction sous l'angle conceptuel, que l'on retrouve en philosophie et en psychanalyse; la traductique, point de jonction entre la théorie de l'information, l'intelligence artificielle, la terminologie, la linguistique et l'informatique; et la traductologie, terme employé par l'auteur pour désigner "la réflexion de la traduction sur ellemême à partir de sa nature d'expérience" $\underline{3}$, sur laquelle je reviendrai.

L'analyse de la traduction, lieu d'une comparaison avec l'original, constitue l'étape la plus importante de la critique des traductions. Elle s'établit sur la base d'une confrontation entre des éléments précis des deux textes d'une part (passages sélectionnés dans l'original, zones textuelles problématiques dans la traduction, etc.), et, d'autre part, entre le projet de traduction et la façon dont il a été réalisé. Elle doit aussi tenir compte des autres traductions du texte, qu'elles soient antérieures, contemporaines, ou encore effectuées dans une autre langue. La deuxième partie du livre, qui se veut une application de la première, donne ainsi à lire successivement le poème "Going to bed" de John Donne, sa traduction en français par Yves Denis et Jean Fuzier (1962), qui sera le plus longuement analysée, puis celles de Philippe de Rothschild (1969), d'Auguste Morel (1924) et enfin la traduction en espagnol d'Octavio Paz (1971). C'est donc encore une fois un exercice de lecture très particulier, la lecture en parallèle de plusieurs textes, qui est proposé. Un travail de lecture devant être complété par un véritable travail d'écriture, étant donné que l'analyse s'adresse, non pas à un professionnel de la traduction, mais bien à un "lecteur de traductions". En ce qui concerne le style, l'auteur insiste sur la clarté de l'exposition, la réflexivité du discours et la nécessité des digressions. Quant à l'évaluation, elle est fondée sur des critères de poéticité et d'éthicité. Il s'agit, d'une part, de questionner la littérarité du texte traduit, et d'autre part, de se demander dans quelle mesure la traduction respecte le texte original. En ce qui concerne la traduction de Denis et Fuzier, l'analyse révèle certaines lacunes, notamment en ce qui a trait à la réflexion sur la poésie anglaise du XVIème siècle, et sur celle de Donne en particulier. Si les traducteurs ont accentué l'aspect érotique du poème, ils l'ont fait au détriment d'éléments importants, comme le "colloquialisme", l'entrelacement de la rhétorique, de la logique et de la poéticité, le lien entre la métaphysique et l'expérience, qui ont complètement disparu lors de la traduction.

Une fois l'analyse de la traduction terminée, on doit procéder à l'étude de sa réception. Il s'agit d'un cas assez particulier de réception étant donné que les commentaires ne portent que très rarement sur la manière dont le texte a été traduit, l'œuvre étant généralement lue par les critiques comme "une œuvre étrangère en français" plutôt que comme une traduction. Enfin, la dernière étape du parcours consiste à élaborer une critique productive de la traduction, à dégager certains principes devant guider une éventuelle re- 
traduction du texte. La critique des traductions n'a donc pas pour but de proposer une nouvelle version du texte mais simplement de donner des éléments de réflexion pour effectuer une traduction plus adéquate que les précédentes.

L'intérêt majeur de cet ouvrage, outre l'étude très minutieuse de la poésie de Donne et de ses traductions, réside certainement dans le fait que l'essai propose un réaménagement de la critique littéraire, considérant le texte traduit comme un type de texte exigeant certes des procédures d'analyse spécifiques, mais devant être, comme tout autre texte littéraire, l'objet de critiques. Si le processus de traduction fait l'objet de recherches dans un domaine théorique précis, éloigné des théories littéraires, l'auteur montre bien que l'étude du texte traduit ne peut se cantonner au domaine de la traduction. Cet ouvrage se distingue donc des réflexions sur la traduction ayant eu cours jusqu'à présent étant donné qu'il propose une nouvelle manière d'aborder le texte traduit, qu'il l'envisage sous une nouvelle perspective. Ce projet était déjà en germe dans L'épreuve de l'étranger, puisqu'il était dit au sujet de l'"analytique de la traduction": "On s'acheminerait par là vers une pratique ouverte, et non plus solitaire, du traduire. Et vers l'institution d'une critique des traductions parallèle et complémentaire à la critique des textes" (p. 19). Seulement, il s'inscrivait dans un projet plus beaucoup plus ambitieux, une théorie qu'il suggérait d'appeler "traductologie" à la fin de l'ouvrage, et où l'analytique de la traduction ne formait que l'un des trois axes principaux, les deux autres étant l'histoire de la traduction et l'éthique de la traduction. Le but était de fonder un espace de réflexion interdisciplinaire, où des disciplines comme la linguistique, les études littéraires, la philosophie, etc., seraient convoquées, et d'élaborer une théorie de la traduction non-ethnocentrique, soucieuse de conserver le caractère étranger du texte original. La "science de la traduction" préconisée possédait également, en plus d'une dimension théorique, une dimension pratique, puisqu'elle devait permettre une scientifisation de la pratique de la traduction. Ces questions seront reprises et développées dans des articles subséquents: dans "La traduction et ses discours", ce sont les les tâches de la traductologie qui sont énumérées; dans "La traduction et la lettre ou l'auberge du lointain" $\underline{4}$, c'est la traduction non-ethnocentrique qui forme plus particulièrement l'objet de l'examen.

Cette préoccupation constante de la lettre de l'original, de son caractère étranger, se retrouve bien évidemment dans le livre Pour une critique des traductions, surtout lors de l'analyse des traductions du poème de Donne, qui fournit à cet effet une très bonne illustration de ce que l'auteur entend par "traduction de la lettre". Opposée à la traduction ethnocentrique, dont l'axiome principal est de "traduire l'œuvre étrangère de façon à ce que l'on ne 'sente' pas la traduction, (...) de façon à donner l'impression que c'est ce que l'auteur aurait écrit s'il avait écrit dans la langue traduisante" $\underline{5}$, la traduction non-ethnocentrique ou "littérale" exige un travail important sur la langue traduisante, une créativité de la part du traducteur. La littéralité

opère au niveau du système de la langue et du texte, au point même où les deux systèmes s'unissent (...) La traduction littérale ne reproduit pas la facticité de l'original, mais la logique qui préside à l'organisation de cette facticité. Elle reproduit cette 
logique, là où la langue traduisante le permet, en ses points nonnormés (que du même coup elle révèle). 6

Il faut noter que cette opposition recoupe celle mise en relief par Schleiermacher dans son article "Des différentes méthodes du traduire" $\underline{7}$, qui lui aussi privilégie la seconde option. L'analyse du texte de Donne met en évidence les mots-clés, les réseaux signifiants sous-jacents, le rythme obtenu par les répétitions, etc. Totalement disparus dans certaines traductions, ces éléments sont à l'origine d'un jeu sur le langage dans d'autres traductions, d'un travail où la langue traduisante est amenée parfois à "se lester d'étrangeté" $\underline{8}$. Si un effet similaire à celui qu'offre la lecture de l'original peut être ainsi créé, c'est parce que le traducteur s'est d'abord fait critique, parce que sa propre interprétation de l'œuvre a guidé son travail de traduction. Le fait de privilégier le travail sur la forme même du texte amène donc en même temps à revaloriser le sujet traducteur.

On comprend dès lors pourquoi la critique des traductions accorde une place si importante au traducteur, à son projet, à l'horizon dans lequel il se situe. Pour comprendre les déformations que l'original a subies, pour reconstituer le parcours interprétatif du traducteur, il importe de prendre en considération le contexte littéraire dans lequel la traduction a été effectuée, les modes de traduction prédominants de l'époque. Quand on connaît l'importance que pouvaient avoir la poésie de Mallarmé et de Valéry pour des traducteurs comme Denis et Fuzier, on comprend que celles-ci aient pu modeler en quelque sorte leur rapport à la poésie de Donne. Voici comment Berman conclut son étude des horizons traductif et poétique de leur traduction:

La double étude d'horizons que nous venons d'effectuer montre que la traduction de Fuzier, "archaïsante" et "valéryenne", est certes une "anomalie" par rapport à la traduction contemporaine de la poésie anglaise, mais qu'elle se relie parfaitement à certaines tendances et de l'horizon poétique, et de l'horizon traductif. Il est donc normal que la majeure partie des critiques l'aient perçu ainsi, et aient pu la trouver comme Poisson "belle et fidèle". (p. 257)

La notion d'horizon, telle qu'elle a été développée entre autres par Jauss $\underline{9}$, est donc appliquée ici à un problème très concret. Il ne s'agit pas seulement de comprendre comment telle œuvre a été reçue à l'époque de sa publication, mais d'évaluer le rôle du contexte culturel dans l'élaboration de la traduction, d'indentifier certains principes interprétatifs ayant guidé les traducteurs dans leur travail sur le texte. Bien entendu, on peut regretter, comme dans le cas de Jauss, que peu de place soit accordée à la première lecture du texte, faite en l'absence de savoirs sur le texte et sur son horizon d'attente. Si, comme l'affirme Berman, la première lecture d'une traduction est d'abord la lecture d'une œuvre étrangère en français, celle-ci possède donc des caractéristiques précises, qui devraient pouvoir être étudiées. Cette première étape de la critique d'une traduction ne mériterait-elle pas de former, à elle seule, l'objet de recherches? Comme le montrent bien le nombre et la diversité des modes de lectures proposés, le critique de traductions est avant quelqu'un qui revient sans cesse sur sa première lecture, celle du texte traduit, en l'enrichissant continuellement afin de 
mettre à jour la nature des liens tissés entre les textes originaux et leurs traductions.

\section{Oeuvres citées}

BERMAN, Antoine, Pour une critique des traductions: John Donne, Paris, Gallimard, coll. Bibliothèque des idées, 1995.

BERMAN, Antoine, "La traduction et ses discours", Meta, vol. 34, no 4, 1989, p. 672-679.

BERMAN, Antoine, "La traduction et la lettre ou l'auberge du lointain", dans A. Berman, éd., Les tours de Babel, Mauvezin, Trans-Europ-Repress, 1985, p. 33-150.

BERMAN, Antoine, L'épreuve de l'étranger. Culture et traduction dans l'Allemagne romantique, Paris, Gallimard, coll. Tel, 1984.

JAUSS, Hans Robert, Pour une herméneutique littéraire, Paris, Gallimard, 1978.

SCHLEIERMACHER, Friedrich, "Des différentes méthodes du traduire", traduit de l'allemand par A. Berman, dans A. Berman, éd., Les tours de Babel, Mauvezin, Trans-Europ-Repress, 1985, p. 278-347.

\section{NOTES}

1Antoine Berman, L'épreuve de l'étranger. Culture et traduction dans

l'Allemagne romantique, Paris, Gallimard, coll. Tel, 1984.

2Antoine Berman, "La traduction et ses discours", Meta, vol. 34, no 4, 1989, p. 672-679.

3ibid., p. 675 .

4Antoine Berman, "La traduction et la lettre ou l'auberge du lointain", dans A. Berman, éd., Les tours de Babel, Mauvezin, Trans-Europ-Repress, 1985, p. 33-150.

5ibid., p. 53.

6ibid., p. 149, l'auteur souligne.

7Friedrich Schleiermacher, "Des différentes méthodes du traduire", traduit de l'allemand par A. Berman, dans A. Berman, éd., Les tours de Babel, Mauvezin, Trans-Europ-Repress, 1985, p. 278-347.

8Berman, L'épreuve de l'étranger, op. cit., p. 18. 
9Hans-Robert Jauss, Pour une herméneutique littéraire, Paris, Gallimard, 1978. 